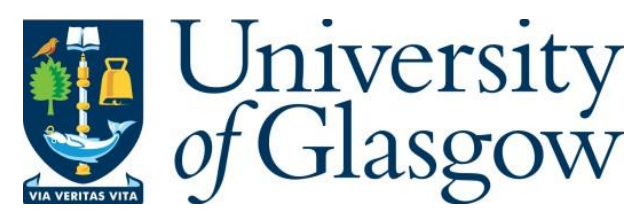

Thomson, N. C. (2018) Bronchial thermoplasty as a treatment for severe asthma: controversies, progress and uncertainties. Expert Review of Respiratory Medicine, 12(4), pp. 269-282.

There may be differences between this version and the published version. You are advised to consult the publisher's version if you wish to cite from it.

http://eprints.gla.ac.uk/158312/

Deposited on: 16 March 2018

Enlighten - Research publications by members of the University of Glasgow http://eprints.gla.ac.uk 
Expert Review of Respiratory Medicine

\title{
Bronchial thermoplasty as a treatment for severe asthma: controversies, progress and uncertainties
}

\author{
Neil C Thomson MD, FRCP, FERS \\ Institute of Infection, Immunity \& Inflammation, \\ University of Glasgow, \\ Glasgow UK
}

Corresponding author

Professor Neil C Thomson, Institute of Infection, Immunity \& Inflammation, University of Glasgow, Glasgow, G12 OYN, UK

Telephone: 44-141-211-1673

Fax: 44-141-211-3464

E-mail: neil.thomson@glasgow.ac.uk

Word count: 6799 words

Financial \& competing interests disclosure

In the last three years Professor Thomson has no conflict of interests to declare 
Title: Bronchial thermoplasty as a treatment for severe asthma: controversies, progress and uncertainties

\begin{abstract}
Introduction

Bronchial thermoplasty is a licensed non-pharmacological treatment for severe asthma.
\end{abstract}

Area covered

This article considers evidence for the efficacy and safety of bronchial thermoplasty from clinical trials and observational studies in clinical practice. Its place in the management of severe asthma, predictors of response and mechanisms of action are reviewed.

\title{
Expert commentary
}

Bronchial thermoplasty improves quality of life and reduces exacerbations in moderate to severe asthma. Morbidity from asthma is increased during treatment. Overall, patients treated in clinical practice have worse baseline characteristics and comparable clinical outcomes to trial data. Follow-up studies provide reassurance on long-term safety. Despite some progress, future 
research needs to investigate uncertainties about predictors of response, mechanism of action and place in management of asthma.

Word count: 121 words

Key words:

Airway smooth muscle; asthma quality of life; bronchial thermoplasty; exacerbations; nonpharmacological intervention; precision medicine; severe asthma; 


\section{Introduction}

Asthma is a chronic inflammatory disease of the airways that affects 300 million people worldwide. Most adults with asthma have mild or moderate disease that potentially can be controlled by inhaled corticosteroids (ICS) alone or in combination with an inhaled long-acting $\aleph_{2}$ agonist (LABA) bronchodilator [1]. Despite the use of current therapies, surveys indicate that many patients have poorly controlled symptoms and experience frequent exacerbations $[2,3]$ due to a range of aggravating factors, such as non-adherence, poor inhaler technique, comorbidities, under treatment and patient characteristics. After identifying and treating contributing factors, a sub-group of patients with difficult-to-control asthma have severe symptomatic disease despite treatment with high-dose ICS and LABA in combination or with continuous or frequent oral corticosteroids [4-7]. The prevalence of difficult-to-control asthma is estimated to be $17.4 \%$ of the total asthmatic population, of whom approximately one fifth have severe refractory disease [8]. Patients with severe asthma have reduced life-expectancy and experience substantial morbidity due to poorly controlled asthma and the adverse effects of high-dose corticosteroids [9]. The financial costs of severe asthma are high due to medications, hospital admissions and time lost from work [10]. Severe asthma persists in most patients, particularly in those with low socioeconomic status and greater comorbidities [11].

Recognition of the heterogeneity of clinical and inflammatory phenotypes of severe asthma has encouraged the development of new treatments, such as monoclonal antibodies targeting immunoglobulin E (anti-lgE) and persistent eosinophilic and type 2 inflammation [12,13]. Anti- 
IgE therapy with omalizumab is a treatment option for severe allergic asthma. Humanized monoclonal antibodies against human interleukin (IL)-5, such as mepolizumab and reslizumab and against the IL-5 receptor $\alpha$, such as benralizumab, were recently approved for add-on maintenance treatment in patients aged 18 years or older with severe eosinophilic asthma. Other therapies targeting type 2 inflammation are in development [13]. Despite this progress, there is a limited understanding of other potentially important phenotypes and endotypes of severe asthma, such as non-eosinophilic asthma and non-type 2 inflammation and there is a bottleneck in the development of novel treatments for certain subtypes of severe asthma [14].

Bronchial thermoplasty is an intervention developed for the treatment of patients with moderate and severe asthma, which involves the delivery of radio frequency energy to the airways [15]. Bronchial thermoplasty is licensed for the treatment of asthma in the United States, Europe, China and in many other countries. The Food \& Drug Administration (FDA) gave premarket approval (PMA) for the Alair ${ }^{\circledR}$ bronchial thermoplasty system as a treatment of severe persistent asthma in patients 18 years and older whose asthma is not well controlled with ICS and LABA in 2010 [16]. In the European Union, the 'Alair' bronchial thermoplasty system has a CE Mark for the treatment of asthma in patients 18 years and older. Controversy exists on the strength of the evidence for the efficacy and safety of bronchial thermoplasty in severe asthma. There are also uncertainties about the effectiveness and safety of bronchial thermoplasty in clinical practice, whether it is possible to predict which patients will obtain clinical benefit from the procedure and its mechanism of action. This review summarizes the pre-clinical and clinical development of bronchial thermoplasty and considers its place in 
management of severe asthma in clinical practice. Recent publications on the potential predictors of response and mechanisms of action are discussed.

\section{Development of bronchial thermoplasty}

Bronchial thermoplasty was developed as a procedure to reduce the amount of airway smooth muscle in severe asthma through the local application of thermal energy to the airways with the aim of improving asthma control [15]. Previously published articles provide detailed reviews of the preclinical and clinical development of bronchial thermoplasty $[15,17-21]$. The key components of the development program are summarized in the following sections.

\section{Preclinical and early clinical studies}

Preclinical studies in dogs demonstrated that bronchial thermoplasty treatment of conducting airways $\geq 3 \mathrm{~mm}$ in diameter, controlled to a temperature of $65^{\circ} \mathrm{C}$, decreased airway responsiveness to methacholine and reduced airway smooth muscle mass up to three years after the procedure [22]. The change in airway responsiveness to methacholine was inversely correlated to the extent of the reduction in airway smooth muscle [22]. Further canine studies showed that bronchial thermoplasty increased airway luminal area, as assessed by changes in computed tomography [23] and in lung inflation pressures [24]. The first study in humans found that bronchial thermoplasty could be safely performed in patients with bronchial carcinoma awaiting lung resection and resulted in reductions of airway smooth muscle mass [25]. In an 
observational study of patients with mild to moderate asthma, bronchial thermoplasty was well tolerated and decreased airway responsiveness to methacholine for at least two years [26]. Based on the findings that bronchial thermoplasty was associated with reductions in airway smooth muscle in dogs and man and that the procedure could be safely performed in patients with asthma, controlled clinical trials of bronchial thermoplasty were initiated in patients with moderate and severe asthma.

\section{Equipment and procedure}

The 'Alair' Bronchial Thermoplasty system includes a radiofrequency generator that regulates the energy applied to the airways and a catheter with an expandable electrode array at its distal end, which is connected to the controller via an integral electrical cable. A footswitch triggers a 10 second period of radiofrequency energy delivered via the catheter. The treatment involves three bronchoscopy sessions with repeated activations of a radiofrequency catheter within the medium and large airways $[27,28]$. Bronchial thermoplasty is performed by pulmonary physicians with experience in bronchoscopy and at facilities equipped to handle respiratory emergencies. Bronchoscopists must undergo special training before undertaking their first procedure as stipulated by the manufactures of the 'Alair' system. Patients receive prophylactic prednisolone or equivalent at a dose of $50 \mathrm{mg}$ per day for the three days before the procedure, the day of the procedure and the day after the procedure. Pre-procedure $\mathrm{FEV}_{1}$ should be greater than or equal to $85 \%$ of a patient's recent value when stable. Patients receive treatment over three outpatient flexible bronchoscopy sessions under conscious sedation 
spaced at approximately three-week intervals, one for each lower lobe and one for both upper lobes. In some centers, bronchial thermoplasty is performed under general anesthesia. Different sedation regimes are used, including mild sedation with midazolam and alfentanil, moderate sedation with target-controlled infusion of propofol and remifentanil or general anesthesia $[27,29]$. The bronchoscope is placed in the distal aspect of the airway being treated and the 'Alair' catheter is advanced under visual guidance. The treatment involves repeated, precise and carefully recorded activations of a radiofrequency catheter within the medium and large airways. A treatment session is typically completed in 45 minutes and around 60 radiofrequency energy treatments delivered to the airways. Before each subsequent session the airways treated in the previous procedure are inspected for persisting inflammation or infection. After each procedure patients are carefully monitored and discharged only when the post-bronchodilator $\mathrm{FEV}_{1}$ is within $80 \%$ of the pre-procedure value and the patient's medical condition is considered stable. The feasibility of hyperpolarized xenon (HXe) magnetic resonance imaging (MRI) as a biomarker to prioritize the order of airway treatment by bronchial thermoplasty is being assessed in patients with severe asthma (ClinicalTrials.gov Identifier: NCT01832363; NCT02263794) [30].

\section{Controlled clinical trials of bronchial thermoplasty}

Evidence for the efficacy and short-term safety of bronchial thermoplasty treatment in patients with moderate to severe asthma is based on the results of three randomized controlled trials $[28,31,32]$. Two trials compared bronchial thermoplasty with usual care, the Asthma 
[30]Intervention Research (AIR) trial [31] and the Research in Severe Asthma (RISA) trial [32], and the third trial (AIR2) compared bronchial thermoplasty with a sham procedure [28].

\section{Efficacy}

In the AIR trial, bronchial thermoplasty reduced the rate of mild exacerbations and improved asthma quality of life questionnaire (AQLQ) scores compared to the usual care group at 12 months [31]. The RISA trial [32], which was primarily designed as a safety study, recruited 34 patients with severe asthma of whom half were taking maintenance oral prednisolone daily, reported that bronchial thermoplasty improved AQLQ scores, asthma control questionnaire (ACQ) scores and rescue medication use compared to usual care at 12 months [32]. There was a trend for more patients in the bronchial thermoplasty group to wean off oral corticosteroids. The pivotal AIR2 trial, which compared bronchial thermoplasty with a sham procedure in 288 adults with moderate to severe asthma, reported that bronchial thermoplasty improved AQLQ scores compared with the sham group (change in AQLQ with bronchial thermoplasty, 1.35 versus 1.16 with sham) [28] (Figure 1). A larger proportion of patients in the bronchial thermoplasty group (79\%) compared with the sham group (64\%) achieved changes in AQLQ of 0.5 or greater in the period from 6 to 12 months after the treatment period [28]. In the posttreatment period (end of treatment period to one year), bronchial thermoplasty compared with the sham procedure reduced the number of severe exacerbations per subject per year by $32 \%$ (0.48 (0.067) bronchial thermoplasty vs $0.70(0.122)$ sham] and emergency department visits by $84 \%$ ( 0.07 bronchial thermoplasty vs. 0.43 sham visits/subject/year) (Figure 1 ). Bronchial 


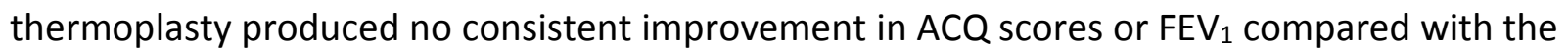
sham subjects [28]. Observational follow-up data beyond one year of patients who completed the AIR2 trial, but not including the sham group, reported that the reduction in the proportion of subjects experiencing severe exacerbations and emergency department visits after bronchial thermoplasty was maintained up to 5 years [33] (Figure 2). An exploratory analysis of the AIR2 results found that a clinically significant improvement in AQLQ score $(\geq 0.5)$ at 1 year in patients treated with bronchial thermoplasty was associated with fewer severe exacerbations and emergency department visits over 5 years compared to those who did not experience clinically significant AQLQ response at year 1 [34]. A Cochrane systematic review of the three randomized controlled trials of bronchial thermoplasty concluded there was a modest clinical benefit in quality of life (mean difference in AQLQ score $0.28,95 \%$ confidence interval $(\mathrm{Cl}) 0.07$ to 0.50 ) and a significant reduction in severe exacerbation rates 12 months after treatment, based on the results of the AIR2 trial [17]. Potential weaknesses in the strength of the evidence from the AIR2 trial have been highlighted, including the proportion of patients recruited to the trial who had severe refractory asthma, whether changes in the primary end point AQLQ were statistically and clinically significant and whether appropriate methods were used to analysis some of the secondary end points [35]. Castro and several other coauthors of the AIR2 trial have provided a rebuttal of the criticisms [36]. In summary, randomized controlled clinical trials of bronchial thermoplasty in patients with moderate and severe asthma show modest improvements in asthma quality of life and reductions in severe exacerbations and emergency department visits. Observational data suggests that these benefits may persist for up to five years. 


\section{Short-term safety}

Bronchial thermoplasty is associated with short-term increases in asthma-related symptoms and hospital admissions for asthma during the treatment period $[28,31,32,37]$. The main adverse effects are wheeze, cough, night awakening and discolored sputum, with most adverse events occurring in the first day after bronchoscopy and resolving within one week. In the AIR2 study, more hospital admissions occurred in the bronchial thermoplasty group $(8.4 \%)$ compared with subjects in the sham group (2\%) during the treatment phase [28]. Over the entire study period (from the day of first bronchoscopy to the 12-month follow-up) there was no difference in the number of respiratory-related hospital admissions per subject in the bronchial thermoplasty group $(0.13,10.5 \%$ of subjects $)$ compared with the sham group $(0.14$ (5.1\% of subjects) [28]. A Cochrane systematic review of the three controlled trial of bronchial thermoplasty reported a 3.5 fold ( $95 \% \mathrm{Cl} 1.26$ to 9.68$)$ greater risk of hospitalization for respiratory adverse events during the treatment period [17]. During the post-treatment period there was no significant difference in the risk of hospital admission (risk ratio 1.12 (95\% $\mathrm{Cl} 0.44$ to 2.85$)$ [17].

\section{Long-term safety}

Observational data on the long-term safety of bronchial thermoplasty is available from the AIR, RISA and AIR2 trials up to five years post bronchial thermoplasty treatment $[33,38,39]$. 
Respiratory adverse events, lung function and rates of hospital admissions or emergency department are unchanged in years two to five following the AIR [38], RISA [39] and AIR2 trials (figure 2) [33]. Serial computed tomography (CT) scans performed in 97 (57\%) of AIR2 subjects treated with bronchial thermoplasty who were followed up to five years showed no clinically significant structural abnormalities to the airways, except for three subjects who had increased or new bronchiectasis compared with the baseline [33]. A criticism of the long-term studies is that only the participants treated with bronchial thermoplasty were followed up after year one in the RISA and AIR2 trials and in the AIR trial, the usual care group were followed-up to the end of year three, whereas the bronchial thermoplasty group were followed for five years. The bronchial thermoplasty at 10 years follow-up or beyond (BT10+) observational study is underway to assess the safety and efficacy of the procedure in 196 patients who previously participated in the three clinical trials (ClinicalTrials.gov Identifier: NCT03243292) [30]. In summary, follow-up data beyond one year provides evidence for the long-term safety of the procedure up to five years in relation to respiratory adverse events reporting, stable lung function and lack of increase in hospital admissions and emergency department visits.

\section{Observational real-life studies in clinical practice}

The introduction of bronchial thermoplasty to clinical practice involves the treatment of reallife patients with moderate and severe asthma, some of whom do not satisfy the inclusion and exclusion criteria used in the clinical trials (Table 1). Information on baseline characteristics and the effectiveness and safety of bronchial thermoplasty in clinical practice is available from 
several case series originating from Australia, Brazil, Canada, France, Japan, Netherlands, United Kingdom and the United States [40-50] (Table 2) and from a United Kingdom national registry [51]. In 2017, the interim three-year results of the PAS2 (Post-FDA Approval Clinical Trial Evaluating Bronchial Thermoplasty in Severe Persistent Asthma) study were published, which is a prospective, open-label, multi-center observational post-market study mandated by the FDA to evaluate the durability of the treatment effect and the short and long-term efficacy and safety of the procedure [52]. The study enrolled 284 participants from 2011 at 27 centres in the United States $(n=23)$ and Canada $(n=4)$ of whom 279 patients received at least one bronchial thermoplasty treatment. The last patient is expected to complete five years of followup in January 2020 (ClinicalTrials.gov Identifier: NCT01350336) [30].

\section{Baseline characteristics}

In general, patients treated with bronchial thermoplasty in clinical practice have more severe disease than those recruited to AIR [31] and AIR2 [28] trials (Table 2). In one case series that compared safety and efficacy outcomes 12-months post-procedure in ten clinic patients with fifteen patients recruited to clinical trials of bronchial thermoplasty at the same center found that asthma severity was greater in clinic patients compared to research patients with 6/10 [60\%) clinic patients at British guideline on the management of asthma Step 5 treatment, compared to $2 / 15(13 \%)$ of the research patients. Baseline asthma control and number of exacerbation was worse in the clinic group [41]. Participants in the PAS2 study compared with those recruited to the AIR2 clinical trial were slightly older, had a higher proportion taking 
maintenance oral corticosteroids ( $18.9 \%$ vs $4.2 \%)$ and had more subjects who experienced severe exacerbations (74\% vs 52\%) and hospitalizations (15.3\% vs $4.2 \%$ ) in the 12 months prior to bronchial thermoplasty. In addition, more subjects in the PAS2 study had chronic sinus disease (30.4\% vs $18.4 \%$ ) and a larger number had severe asthma (94.7\% vs $82.1 \%$ )[52]. The British Thoracic Society (BTS) Difficult Asthma Registry and Hospital Episodes Statistics database of 59 patient with severe refractory asthma undergoing bronchial thermoplasty in clinical practice in the UK between 2011 and 2015 reported that bronchial thermoplasty patients were on average, older, had worse baseline $\mathrm{FEV}_{1}$ and lower AQLQ scores compared with published clinical trials [51].

\section{Effectiveness and safety}

Despite the limitations of an observational study design, the interim-analysis of PAS2 study suggests that improvements in efficacy outcomes in the PAS2 populations and AIR2 participants were similar [52]. At three years post-treatment, the proportion of people with severe exacerbations, emergency department visits and hospitalizations was reduced by $45 \%, 55 \%$, and $40 \%$ respectively when compared to the 12 months prior to treatment, which were comparable to reductions of $37 \%, 72 \%$ and $25 \%$ respectively reported in AIR2 [28]. Pre- and post-bronchodilator spirometry was unchanged over the 3 years of follow-up after bronchial thermoplasty. Previous published information from observational studies on the effectiveness of bronchial thermoplasty for severe asthma in real-life patients have reported improvement in 
AQLQ scores, reductions in exacerbations and/or a step-down in treatment in $50 \%$ to $75 \%$ of patients undergoing the procedure (Table 2).

Respiratory-related serious adverse effects during the treatment phase with bronchial thermoplasty (first bronchial thermoplasty treatment to 6 weeks after last procedure) were greater in the PAS2 study [52] compared to the AIR2 [28] for severe exacerbations (55.8\% vs. $40.5 \%$ respectively) and emergency room visits ( $15.8 \%$ vs. $5.3 \%$ respectively), whereas emergency respiratory hospital readmission rates (within 30 days) of bronchial thermoplasty were similar (13.2\% vs $8.4 \%$ respectively). Previous observational studies report hospital admissions ranging from $10 \%$ [45] to $30 \%$ [41] during the treatment phase. The BTS Difficult Asthma Registry recorded a hospital admission rate of $11.8 \%$ in 59 cases undergoing bronchial thermoplasty in the United Kingdom [51]. Patients have been reported to safely undergo bronchial thermoplasty with baseline characteristics that would have excluded them from enrolment to the AIR2 trial including those with prebronchodilator $\mathrm{FEV}_{1}<60 \%$ predicted $[40,48]$ and who were former smokers with more than 10 pack years total smoking history [42].

CT imaging performed the day after bronchial thermoplasty in 13 patients with severe asthma demonstrated pulmonary peribronchial consolidations and ground-glass opacities in all treated lobes and mild involvement of an adjacent untreated lobe in $32 \%$ of cases [53]. The CT changes were not associated with respiratory symptoms and deceased or disappeared without treatment after one month. Another study of 12 patients with severe asthma found CT changes shortly after bronchial thermoplasty that included peribronchial consolidations with 
surrounding ground glass opacities (94\%), atelectasis (38\%), partial bronchial occlusions (63\%), and bronchial dilatations (19\%) [54]. Follow-up CT scans at 6 months showed resolution of the changes except for an area of focal bronchiectasis in a single airway. In summary, these studies indicate that bronchial thermoplasty-induced acute CT pulmonary peribronchial consolidation frequently occurs and that these changes resolve in most patients within one to six months.

The strength of the evidence provided from observational studies on the effectiveness and safety of bronchial thermoplasty in real-life patients has several weaknesses including the lack of control groups that was not treated with bronchial thermoplasty and it is unclear whether improvements in clinical outcomes differ from usual care [55]. The PAS2 study population is described as real-world, although the most severe patients seen in clinical practice were excluded, such as those with a baseline forced expiratory volume in 1 second $\left(\mathrm{FEV}_{1}\right)<60 \%$, more than 3 hospitalizations, 4 or more courses of systemic corticosteroids in the last 12 months and oral corticosteroids maintenance dose $>10 \mathrm{mg}$ daily (Table 1 ).

In summary, real-life patients treated with bronchial thermoplasty in clinical practice tend to have more severe disease than those treated in clinical trials of the procedure. The interimanalysis of PAS2 suggests that reductions in exacerbations rates and emergency department visits at 3-year post bronchial thermoplasty in patients with severe asthma are comparable to those reported in the AIR2 trial, although adverse respiratory effects occur more frequently during the treatment period. Observational studies report that bronchial thermoplasty is effective in one half to three-quarters of real-life patients with severe asthma. The procedure 
can be safely performed in clinical practice although peri-procedure adverse effects are commoner than reported in clinical trials of bronchial thermoplasty.

\section{Important unresolved issues about bronchial thermoplasty}

Unresolved issues about bronchial thermoplasty include uncertainties about its place in the management of severe asthma, predictors of a beneficial response and mechanism(s) of action.

\section{Place of bronchial thermoplasty in the management of severe asthma}

The Global Initiative for Asthma (GINA) guideline for adults and adolescents with asthma recommends a step-wise approach to control symptoms and minimize future risk and to reduce treatment burden, especially from oral corticosteroid use in patients with severe disease [1]. Patients with severe asthma (step 5) who have persistent symptoms and exacerbations should be assessed by a specialist in severe asthma to confirm the correct diagnosis and to address aggravating factors, such as non-adherence, poor inhaler technique and co-morbidities. Add-on treatment options for patients with uncontrolled asthma despite receiving high dose ICS plus LABA and the antimuscarinic agonist tiotropium include biologics, such as anti-IgE therapy and anti-IL-5 therapy and low dose oral corticosteroid. Precision medicine is increasingly being used in the management of severe asthma due to the availability of biomarkers that can predict a positive response to specific interventions. For example, elevated total serum IgE levels, as well as biomarkers of type 2 inflammation [56], help select patients with severe allergic asthma 
suitable for anti-lgE therapy with omalizumab and a raised blood eosinophil count identifies patients suitable for therapy with monoclonal antibodies targeting IL-5 with subcutaneous mepolizumab [57], intravenous reslizumab [58] or subcutaneous benralizumab [59]. The GINA guideline considers bronchial thermoplasty as a potential treatment option at step 5 in some countries for selected adults with severe asthma whose asthma remains uncontrolled despite optimized therapy and review by an asthma specialist [1]. Evidence is considered limited (Evidence B) and long-term effects compared to controls are not known. Longer-term safety follow-up of larger number of active and control patients is required to assess effectiveness and safety [1]. The International ERS/ATS Guidelines on Definition, Evaluation and Treatment of Severe Asthma recommend that bronchial thermoplasty is performed in adults with severe asthma only in the context of an Institutional Review Board approved independent systematic registry or a clinical study [5]. The British guideline on the management of asthma recommends that bronchial thermoplasty is a modestly effective treatment option for selected patients with moderate to severe asthma who have poorly controlled asthma despite maximal therapy [60].

Patients selected for bronchial thermoplasty in clinical practice are more likely to have clinical features of more severe disease than those recruited to clinical trials of the procedure. Several authors have suggested that bronchial thermoplasty should be considered for patients with severe asthma associated with non-type 2 inflammation and non-eosinophilic inflammation $[61,62]$. To date, there are no clinical trials that that have directly compared the effectiveness of bronchial thermoplasty in severe asthma with biological agents, such as omalizumab or antiIL-5 therapies. Possible biomarkers that predict a favourable response to bronchial 
thermoplasty are being assessed and are reviewed in the next section. The balance of risks and benefits of bronchial thermoplasty treatment should be discussed with patients. Patients with a pacemaker, internal defibrillator, or other implantable electronic device, known sensitivity to medications required to perform bronchoscopy or who have previously received treatment with bronchial thermoplasty should not receive bronchial thermoplasty treatment.

Several studies have estimated the cost-effectiveness of bronchial thermoplasty compared to usual care in patients with severe asthma treated in different health care systems. In the United States, bronchial thermoplasty is estimated to be cost-effective in patients with severe uncontrolled asthma who have a high risk of exacerbations [63] or in patients with moderate to severe allergic asthma [64], whereas in Singapore, the procedure is not cost-effective compared to optimized usual care [65]. The likelihood of bronchial thermoplasty being a cost-effective treatment for severe asthma in a health care system are greater if the costs of hospitalization and emergency department are high and the costs of the procedure are low [65]. Other factors that influence the cost-effectiveness of bronchial thermoplasty are a patient's risk of a future exacerbation, the duration of follow-up after treatment and the health systems threshold in dollars for the willingness-to-pay per quality-adjusted life years (QALY). An indirect comparison of bronchial thermoplasty with omalizumab suggested that there was more than a $60 \%$ chance that bronchial thermoplasty became cost-effective relative to omalizumab and standard therapy at the willingness-to-pay of $\$ 100,000$ per QALY in patients with moderate-to-severe allergic asthma in the United States [64,66]. Another study, using data from the AIR2 trial of bronchial thermoplasty and two placebo-controlled trials of omalizumab (INNOVATE [67] and 
EXTRA [68]), reported broadly similar clinical outcomes with the two treatments, including severe exacerbations, emergency department visits, and hospital admissions [66]. Asthmarelated emergency department visits were less for the post-treatment period of the AIR2 trial compared to omalizumab and severe exacerbations were less for omalizumab compared to the total duration of the AIR2 study that included the increase in exacerbations during the pertreatment period [66]. Future studies should assess the cost effectiveness of bronchial thermoplasty with the long-term use of biologics or maintenance oral corticosteroids.

In summary, bronchial thermoplasty is a treatment option for selected patients with moderate to severe asthma who have poorly controlled asthma despite maximal therapy. There is a lack of evidence about which subphenotype(s) of severe asthma should be treated with bronchial thermoplasty compared to other treatments, such as biological agents. Some experts have recommended that bronchial thermoplasty should be used mainly in patients with severe asthma associated with non-type 2 inflammation and non-eosinophilic inflammation or as a second-line alternative therapy for patient unresponsive to currently licenced biologics.

\section{Predicting a beneficial response to bronchial thermoplasty}

There is limited information on factors that can identify patients with severe asthma who will obtain clinical benefits from bronchial thermoplasty, particularly alongside the use of new biologic therapies. Clinical and physiological variables, such as a history of allergy [33] or bronchodilator reversibility [45] are not predictive. Preliminary findings from a United States 
study of 47 patients with severe asthma suggested that patients with early onset atopic severe asthma tend to respond better than other phenotypes to bronchial thermoplasty [49]. Another preliminary report suggested that shorter duration of asthma, lower baseline AQLQ score and higher continuous maintenance oral corticosteroid dose may be predictive [69] although further confirmatory evidence is awaited. Future analysis of the large PAS2 population of severe asthma may identify potential clinical predictors of response [52].

Using data from 24 consecutive cases of severe asthma treated with bronchial thermoplasty at three Australian centres, Langton and colleagues [70] found that the number of activations delivered to the airways was lower in non-responders compared to responders $(139 \pm 11$ activations versus $221 \pm 45$ activations respectively) and the improvement in ACQ score correlated with a greater number of activations. In another study, the cumulative number of activations administered during three sessions of bronchial thermoplasty were not associated with bronchial biopsy histological changes induced by the procedure [44].

Biomarkers of airway inflammation, such as sputum differential cell count and exhaled nitric oxide were not measured in the clinical trials of bronchial thermoplasty and to date it is unknown whether these variables are predictive of a beneficial response to bronchial thermoplasty. One preliminary report found that responders to bronchial thermoplasty had lower serum periostin levels and exhaled nitic oxide (FeNO) levels compared to non-responders [71]. To date, preliminary findings from bronchial biopsy studies of patients undergoing bronchial thermoplasty have found that the amount of airway smooth muscle at baseline does 
not predict a beneficial response [44,50] (ClinicalTrials.gov Identifier: NCT01777360;

NCT02225392) [30]. Imaging of the airways by optical coherence tomography [72], CT [47] and endobronchial ultrasound are potential methods to identify predictors of response to bronchial thermoplasty. Optical coherence tomography of the airway performed 2-years after bronchial thermoplasty in two patients reported a decrease in airway wall thickness in one patient who responded to the procedure and an increase in the other demonstrated differences in airway wall features between the responder and non-responder prior to treatment [72]. The BTR (Biopredictors of Bronchial Thermoplasty Response in Patients With Severe Refractory Asthma) study is underway to determine whether baseline clinical, physiologic, biologic and imaging markers can identify those patients with severe asthma who will obtain greatest benefit from bronchial thermoplasty (ClinicalTrials.gov Identifier: NCT01185275) [30].

\section{Mechanism of action of bronchial thermoplasty}

Several mechanisms of action of bronchial thermoplasty have been proposed, including alterations to the structure or function of airway smooth muscle and/or other airway cells and structures including epithelial cells, nerves, extracellular matrix and inflammatory cells [73].

\section{Reduced airway smooth muscle mass}

Increased airway smooth muscle mass in asthma [74], particularly in severe disease [75], may contribute to impaired lung function, airway hyperresponsiveness and poor symptom control 
$[76,77]$. Bronchial thermoplasty reduces the amount of airway smooth muscle in experimental animals [22] and in patients with bronchial carcinoma awaiting lung resection [25]. Bronchial biopsy studies in asthma have confirmed that airway smooth muscle mass is reduced by bronchial thermoplasty (Figure 3) [42-44,78,79]. Preliminary findings from the TASMA (Unravelling Targets of Therapy in Bronchial Thermoplasty in Severe Asthma) study, in which patients were randomized to either bronchial thermoplasty or delayed treatment after six months, reported that airway smooth muscle mass decreased after bronchial thermoplasty, but did not change in the controls or the untreated right middle lobe (ClinicalTrials.gov Identifier: NCT02225392) [30,80]. Several studies have investigated whether structural changes to the airway are associated with improvements in clinical outcomes. In the ASMATHERM (Bicentric Prospective Study, Evaluating Bronchial THERMOPLASTY in Patients Presenting Severe Uncontrolled Asthma) study of 15 patients with severe asthma, Pretolani and colleagues [44] found that the reduction in airway smooth muscle mass correlated with the improvement in asthma control and quality of life and decrease in severe exacerbations at 1 year after bronchial thermoplasty (ClinicalTrials.gov Identifier: NCT01777360) [30]. Another study found no association between reduction in airway smooth muscle mass or reticular basement membrane thickness and clinical improvements after bronchial thermoplasty $[42,43]$. Imaging techniques have been used to investigate the effects of bronchial thermoplasty on airway thickness. A study of 26 patients with severe asthma showed improvements in CT airway wall thickness and air trapping one year after bronchial thermoplasty [47]. Another study using three-dimensional airway analysis of CT scans performed before and after bronchial thermoplasty in one patient demonstrated dilation of the bronchial lumen and decreased bronchial wall thickness [81]. 
Optical coherence tomography of the airway performed 2-yeasr after bronchial thermoplasty in two patients reported a decrease in airway wall thickness in a patient who responded to the procedure and an increase in the non-responder [72]. In summary, these findings suggest that bronchial thermoplasty is likely to decrease airway smooth muscle mass in asthma, but they do not establish whether the clinical benefits are due to this mechanism.

\section{Altered airway smooth muscle function}

Bronchial thermoplasty could alter airway function by reducing contractility of airway smooth muscle [82]. The contraction of bovine airway smooth muscle to acetylcholine is inhibited at temperatures greater than $55^{\circ} \mathrm{C}$ and actin-myosin interactions are similarly temperature sensitive. Whether the acute effect of temperatures in vitro smooth muscle responsiveness translates to long-term reductions in airway contractility after bronchial thermoplasty is unclear. Bronchial thermoplasty may also alter airway function by stiffening the airway wall [22] or by reducing the secretion of pro-inflammatory mediators from airway smooth muscle cells [77].

Altered airway epithelial cells, glands, nerves, extracellular matrix components

Bronchial thermoplasty might improve clinical outcomes in asthma by altering the structure and/or function of airway epithelial cells, glands, nerves or extracellular matrix components. In one observational study, bronchial thermoplasty reduced subepithelial basement membrane 
thickening, submucosal nerves, airway smooth muscle-associated nerves and epithelial neuroendocrine cells (Figure 3) [44]. No effect was found on subepithelial mucous glands or the epithelial structure, including the proportion of regenerating bronchial epithelium or normal stratified columnar, metaplastic, or squamous epithelium or goblet cell hypertrophy or hyperplasia at one year after the procedure [44]. In another study, bronchial thermoplasty decreased type I collagen deposition and reticular basement membrane thickness [42,43]. A preliminary report from this latter study found that the bronchial epithelium showed increased cell proliferation and basal progenitor cells numbers and reduced MUC5AC mucin expression [83] that correlated with the reduction in the rates of severe exacerbations during the $\geq 27$ month post-bronchial thermoplasty period [83]. The authors speculate that the long-term benefits from bronchial thermoplasty may be due, at least in part, to an improvement in the structure of the bronchial epithelium [83]. Another preliminary study reported increased epithelial integrity six months following bronchial thermoplasty [84]. In a study of 15 patients with severe asthma, Pretonali and colleagues [44] found that the reduction in submucosal nerves and number of epithelial neuroendocrine cells correlated with the decrease in severe exacerbations at 1 year after bronchial thermoplasty. The reduction in the number of epithelial neuroendocrine cells also correlated with improvement in asthma control test (ACT) and AQLQ scores [44]. In summary, preliminary data suggests that airway epithelium and nerves may be involved in the mode of action of bronchial thermoplasty.

\section{Inflammation}


Information is limited about the effects of bronchial thermoplasty on biomarkers of inflammation in asthma. A recent study in 11 patients with severe asthma reported a decrease in bronchoalveolar lavage concentrations of transforming growth factor $\beta 1$ and chemokine (C-C motif) ligand 5 (CCL5) at 6 weeks after bronchial thermoplasty whereas IL-4, IL-5, and IL-17 levels were unaffected [79]. A small retrospective uncontrolled study of 15 patients undergoing bronchial thermoplasty reported a reduction in peripheral blood eosinophil count at one year after the procedure, although the mechanism is uncertain [85]. Bronchial mucosal eosinophils are not reduced after bronchial thermoplasty, suggesting that the clinical benefits of bronchial thermoplasty are not due to attenuated airway eosinophilic inflammation [44]. A preliminary report on the effects of bronchial thermoplasty on blood gene expression found that multiple pathways decrease significantly including pathways associated with eosinophilic inflammation, T cell activation, and neuronal function [86]. The authors speculate that the beneficial effects of bronchial thermoplasty may be in part due to modulation of allergic inflammation and neuronal control of airway function [86]

\section{Site of action in the airways}

Small airway dysfunction is a feature of severe asthma [87]. It is uncertain whether bronchial thermoplasty influences the structure or function of peripheral airways $(<3 \mathrm{~mm})$, particularly as treatment is applied to large airways (>3 mm). CT imaging performed shortly after bronchial thermoplasty shows acute peribronchial consolidations adjacent to treated airways that is likely to involve the peripheral airways $[53,54]$. Preliminary findings from small observational studies 
in patients with severe asthma pre- and post-bronchial thermoplasty found improvements in measurements of small airway dysfunction assessed by the forced oscillatory technique and by measurement of static and dynamic lung compliance and lung resistance $[88,89]$. Taken together, this recent preliminary data suggests that some of the clinical benefits of bronchial thermoplasty may be due to effects on the small airways [90].

\section{Placebo effect}

Sham interventions are associated with considerable placebo effects in medical device trials [91]. In the AIR2 trial, the sham-controlled arm was associated with increased AQLQ scores, although the bronchial thermoplasty produced greater improvements in AQLQ scores, and was associated with a reduction in severe exacerbations and emergency room visits [28]. Some of the clinical benefits from bronchial thermoplasty in asthma may be due to a placebo effect.

\section{Expert commentary}

Severe refractory asthma, although uncommon, causes considerable morbidity and mortality and generates high health care costs. Until recently, there were limited advances in the therapies available for severe asthma. The recognition of the heterogeneity of clinical and inflammatory phenotypes of severe asthma has resulted in the development and licencing of new biological therapies targeting specific immunological pathways [1]. Bronchial thermoplasty, which involves the delivery of radio frequency energy to the airways, is a non-pharmacological 
intervention developed for the treatment of severe asthma, which has been approved for use in many countries world-wide. The main evidence for the effectiveness of bronchial thermoplasty for the treatment of severe asthma is based on the results of three randomized controlled trials, only one of which compared the procedure with a sham control (AIR2) [28]. The methods of analysis and interpretation of the results of these trials has generated considerable debate and the lack of inclusion of the sham-control group or usual care groups in the long-term follow-up safety studies has been criticised. Biologics and small-molecule drugs for asthma require evidence of efficacy and safety in replicate phase 3 clinical trials before being considered for regulatory approval, whereas a medical device, such as that used to administer bronchial thermoplasty required less stringent criteria of efficacy for approval. A replicate of the AIR2 trial would have been helpful in assessing the effectiveness of bronchial thermoplasty. Despite the weaknesses in the evidence for the efficacy and safety of bronchial thermoplasty from clinical trials, overall, these studies shown that bronchial thermoplasty produces modest improvements in asthma quality of life and reductions in the frequency of severe exacerbations. During the treatment period, bronchial thermoplasty is associated with increased asthma-related symptoms and hospital admissions for asthma. Long-term follow-up studies have not reported any serious adverse effects.

The introduction of bronchial thermoplasty to clinical practice has resulted in its use in real-life patients, some of whom do not satisfy the inclusion and exclusion criteria for the clinical trials. Observational studies indicate that real-life patients are more likely to have clinical features of severe disease than AIR2 participants. The efficacy and safety of bronchial thermoplasty in real- 
life studies is comparable to AIR2, although a major weakness of the data is the lack of a control group that was not treated with bronchial thermoplasty. The PAS2 observational study, which provides the largest data on real-life patients undergoing bronchial thermoplasty, offers reassurance on the long-term safety of bronchial thermoplasty in clinical practice, although the interim results await confirmation when the PAS2 cohort reach 5-years of follow-up in 2020. It is important that the short-term and long-term effectiveness and safety of bronchial thermoplasty in real-world populations continues to be recorded in national [51] and international databases (ClinicalTrials.gov Identifier: NCT02104856) [30].

The place of bronchial thermoplasty in the management of severe asthma is uncertain. Several subtypes or phenotypes of severe asthma are recognized, based on clinical, physiological or immunological variables, and these are used to classify asthma and to predict response to therapy [12,13]. Type 2 inflammation is closely associated with eosinophilic inflammation and predicts a favourable therapeutic response to biological agents targeting the $\mathrm{Th}_{2}$ pathway including interleukin (IL)s 4, IL-5, IL-13 and anti-thymic stromal lymphopoietin (TLSP). Noneosinophilic inflammation and non-type 2 inflammation are poorly responsive to current therapies including corticosteroids [12,92]. An algorithm for the use of bronchial thermoplasty and biologics in management of severe asthma in clinical practice is outlined in Figure 4. Currently, the suggestion that bronchial thermoplasty should be considered for patients with severe asthma associated with non-type 2 inflammation and non-eosinophilic inflammation is based on the availability of effective biologics therapies for type 2 inflammation and eosinophilic inflammation, rather than because of evidence that the efficacy of bronchial 
thermoplasty is related to specific inflammatory phenotypes of severe asthma. Clinical trials that directly compared bronchial thermoplasty, biologics such as omalizumab, anti-IL-5 monoclonal antibodies and novel small molecule drugs [93] in severe asthma could help establish validated treatment pathways.

Potential clinical or inflammatory biomarkers that might predict a beneficial response to bronchial thermoplasty, such as early age of onset of asthma, non-type 2 inflammation, increased airway smooth muscle mass or CT or optical coherence tomography images and number of activations delivered to the airways need to be validated in large populations of patients with severe asthma. Studies underway may provide insights into predictors of response to bronchial thermoplasty [30]. Possible modes of action of bronchial thermoplasty under investigation include reduced airway smooth muscle mass or function, alterations to the structure and/or function of airway epithelial cells, nerves or extracellular matrix components. Studies are underway to investigate the mode of action of bronchial thermoplasty and to identify biomarkers that predict a beneficial response [30].

\section{Five-year review}

The strict eligibility criteria used in many clinical trials of therapies for severe asthma exclude over $90 \%$ of real-life patients [94] suggesting that guideline recommendations may not be applicable to all patients with severe asthma seen in daily practice. Ideally, new clinical trials will provide data on the effectiveness of bronchial thermoplasty treatment for severe asthma, 
particularly in high-risk patients, compared to a sham procedure or to new biologics.

Unfortunately, new large clinical trials may not be undertaken due to their high costs and potential difficulties undertaking comparative clinical studies using treatments developed by different pharmaceutical and device companies. Over the next five years, new data on the effectiveness and safety of bronchial thermoplasty and the identification of predictors of response is likely to come from observational studies in real-world populations of severe asthma. Greater understanding of the sub-phenotypes of severe asthma and mode of action of bronchial thermoplasty may aid the introduction of a precision medicine approach to the use of bronchial thermoplasty in the management of severe asthma. Alternative approaches to target airway smooth muscle may be developed for clinical use, such by electroporation ablation of bronchial smooth muscle through the delivery of non-thermal energy via an endoscope [95]. 


\section{Key issues}

- Bronchial thermoplasty is an intervention developed for the treatment of patients with severe asthma, which involves the delivery of radio frequency energy to the airways.

- Randomized controlled clinical trials of bronchial thermoplasty in patients with moderate and severe asthma show modest improvements in asthma quality of life and reductions in severe exacerbations and emergency department visits.

- Morbidity from asthma is increased during treatment. Follow-up data beyond one year have not reported any serious adverse effects up to five years

- Observational studies report that bronchial thermoplasty is effective in one half to threequarters of real-life patients with severe asthma. The procedure can be safely performed in clinical practice although per-procedure adverse effects are commoner than reported in clinical trials of bronchial thermoplasty.

- Bronchial thermoplasty is a treatment option for selected patients with moderate to severe asthma who have poorly controlled asthma despite maximal therapy. It is not known which subphenotype(s) of severe asthma should be treated with bronchial thermoplasty compared to other treatments, such as biological agents. Currently, bronchial thermoplasty is mainly used in patients with severe asthma associated with non-type 2 inflammation and non-eosinophilic inflammation or as a second-line alternative therapy for patient unresponsive to currently licenced biologics. 
- Preliminary data suggest that certain clinical variables or inflammatory biomarkers might predict a beneficial response to bronchial thermoplasty, but these factors need to be validated in large populations of patients with severe asthma.

- Modes of action of bronchial thermoplasty under investigation include reduced airway smooth muscle mass or function, alterations to the structure and/or function of airway epithelial cells, nerves or extracellular matrix components.

- Future research needs to investigate uncertainties about predictors of response, mechanism of action and the place in management of bronchial thermoplasty for severe asthma. 


\section{Key references}

Papers of special note have been highlighted as:

* of interest

** of considerable interest

1. GINA Report, Global Strategy for Asthma Management and Prevention http://www.ginasthma.com Accessed 4 ${ }^{\text {th }}$ January 2018.

2. Demoly P, Annunziata K, Gubba E, Adamek L. Repeated cross-sectional survey of patient-reported asthma control in Europe in the past 5 years. Eur Respir Review, 21(123), 66-74 (2012).

3. Price $D$, Fletcher $M$, van der Molen T. Asthma control and management in 8,000 European patients: the REcognise Asthma and LInk to Symptoms and Experience (REALISE) survey. NPJ Prim Care Respir Med, 24, 14009 (2014).

4. Bel EH, Sousa A, Fleming $L$ et al. Diagnosis and definition of severe refractory asthma: an international consensus statement from the Innovative Medicine Initiative (IMI). Thorax, 66(10), 910-917 (2011).

5. Chung KF, Wenzel SE, Brozek JL et al. International ERS/ATS guidelines on definition, evaluation and treatment of severe asthma. Eur Respir J, 43(2), 343-373 (2014).

6. Heaney LG, Brightling CE, Menzies-Gow A, Stevenson M, Niven RM, on behalf of the British Thoracic Society Difficult Asthma N. Refractory asthma in the UK: cross-sectional findings from a UK multicentre registry. Thorax, 65, 787-794 (2010).

7. Wark PAB, Hew M, Maltby S, McDonald VM, Gibson PG. Diagnosis and investigation in the severe asthma clinic. Exp Rev Respir Med, 10(5), 491-503 (2016).

8. Hekking P-PW, Wener RR, Amelink M, Zwinderman AH, Bouvy ML, Bel EH. The prevalence of severe refractory asthma. J Allergy Clin Immunol, 135(4), 896-902 (2015).

9. Sweeney J, Patterson CC, Menzies-Gow A et al. Comorbidity in severe asthma requiring systemic corticosteroid therapy: cross-sectional data from the Optimum Patient Care 
Research Database and the British Thoracic Difficult Asthma Registry. Thorax, 71(4), 339-346 (2016).

10. O'Neill S, Sweeney J, Patterson CC et al. The cost of treating severe refractory asthma in the UK: an economic analysis from the British Thoracic Society Difficult Asthma Registry. Thorax, 70(4), 376-378 (2015).

11. Chen W, Marra CA, Lynd LD, FitzGerald JM, Zafari Z, Sadatsafavi M. The natural history of severe asthma and influences of early risk factors: a population-based cohort study. Thorax, 71(3), 267-275 (2016).

12. Fahy JV. Type 2 inflammation in asthma - present in most, absent in many. Nat Rev Immunol, 15(1), 57-65 (2015).

13. Ray A, Raundhal M, Oriss TB, Ray P, Wenzel SE. Current concepts of severe asthma. J Clin Invest, 126(7), 2394-2403 (2016).

14. Barnes PJ, Bonini S, Seeger W, Belvisi MG, Ward B, Holmes A. Barriers to new drug development in respiratory disease. Eur Respir J, 45(5), 1197-1207 (2015).

15. * Cox PG, Miller J, Mitzner W, Leff AR. Radiofrequency ablation of airway smooth muscle for sustained treatment of asthma: preliminary investigations. Eur Respir J, 24(4), 659663 (2004).

The first description of the development of bronchial thermoplasty as procedure to reduce the amount of airway smooth muscle in severe asthma through the local application of thermal energy to the airways with the aim of improving asthma control

16. U.S. Food and Drug Administration. In: Alair Bronchial Thermoplasty System: Alair Catheter and Alair RF Controller (Ed.^(Eds) (https://www.accessdata.fda.gov/cdrh docs/pdf8/p080032a.pdf, 2010 Accessed $4^{\text {th }}$ January 2018

17. * Torrego A, Solà I, Munoz A et al. Bronchial thermoplasty for moderate or severe persistent asthma in adults. Cochrane Database Syst Rev, Issue 3. Art. No.: CD009910. DOI: 10.1002/14651858.CD009910.pub2. (2014).

A Cochrane systematic review of the three randomized controlled trials of bronchial thermoplasty concluded there was a modest clinical benefit in asthma quality of life and 
a significant reduction in severe exacerbation rates 12 months after treatment. There was a 3.5 fold greater risk of hospitalization for respiratory adverse events during the treatment period.

18. Thomson NC, Bicknell S, Chaudhuri R. Bronchial thermoplasty for severe asthma. Curr Opin Allergy Clin Immunol, 12(3), 241-248 (2012).

19. Dombret M-C, Alagha K, Philippe Boulet L et al. Bronchial thermoplasty: a new therapeutic option for the treatment of severe, uncontrolled asthma in adults. Eur Respir Rev, 23(134), 510-518 (2014).

20. Sheshadri A, McKenzie M, Castro M. Critical Review of Bronchial Thermoplasty: Where Should It Fit into Asthma Therapy? Curr Allergy Asthma Rep, 14(11), 470 (2014).

21. Laxmanan B, Egressy K, Murgu SD, White SR, Hogarth DK. Advances in bronchial thermoplasty. Chest, 150(3), 694-704 (2016).

22. Danek CJ, Lombard CM, Dungworth DL et al. Reduction in airway hyperresponsiveness to methacholine by the application of RF energy in dogs. J App/ Physiol, 97(5), 1946-1953 (2004).

23. Brown RH, Wizeman W, Danek C, Mitzner W. In vivo evaluation of the effectiveness of bronchial thermoplasty with computed tomography. J Appl Physiol, 98(5), 1603-1606 (2005).

24. Brown RH, Wizeman W, Danek C, Mitzner W. Effect of bronchial thermoplasty on airway distensibility. Eur Respir J, 26(2), 277-282 (2005).

25. Miller JD, Cox G, Vincic L, Lombard CM, Loomas BE, Danek CJ. A Prospective Feasibility Study of Bronchial Thermoplasty in the Human Airway. Chest, 127(6), 1999-2006 (2005).

26. Cox G, Miller JD, McWilliams A, FitzGerald JM, Lam S. Bronchial Thermoplasty for Asthma. Am J Respir Crit Care Med, 173(9), 965-969 (2006).

27. Bicknell S, Chaudhuri R, Thomson NC. How to: Bronchial thermoplasty in asthma. Breathe, 10(1), 48-59 (2014).

28. ** Castro M, Rubin AS, Laviolette M et al. Effectiveness and Safety of Bronchial Thermoplasty in the Treatment of Severe Asthma: A Multicenter, Randomized, Double- 
Blind, Sham-Controlled Clinical Trial. Am. J. Respir. Crit. Care Med., 181(2), 116-124 (2010).

The pivotal AIR2 trial compared bronchial thermoplasty with a sham procedure in 288 adults with moderate to severe asthma and reported in the post treatment period that bronchial thermoplasty improved AQLQ scores and reduced the number of severe exacerbation compared with the sham group.

29. d'Hooghe JNS, Eberl S, Annema JT, Bonta PI. Propofol and Remifentanil Sedation for Bronchial Thermoplasty: A Prospective Cohort Trial. Respiration, 93(1), 58-64 (2017).

30. U.S. National Institutes of Health, 2018 https://clinicaltrials.gov/. Accessed $4^{\text {th }}$ January 2018

31. Cox G, Thomson NC, Rubin AS et al. Asthma Control during the Year after Bronchial Thermoplasty. N Eng J Med, 356(13), 1327-1337 (2007).

32. Pavord ID, Cox G, Thomson NC et al. Safety and Efficacy of Bronchial Thermoplasty in Symptomatic, Severe Asthma. Am J Respir Crit Care Med, 176(12), 1185-1191 (2007).

33. *Wechsler ME, Laviolette M, Rubin AS et al. Bronchial thermoplasty: Long-term safety and effectiveness in patients with severe persistent asthma. J Allergy Clin Immunol, 132, 1295-1302 (2013).

Observational data on the long-term safety of bronchial thermoplasty in the AIR2 trial up to 5 years post bronchial thermoplasty treatment, but not including follow-up of the sham group. The rate of respiratory adverse events, lung function and rate of hospital admissions or emergency department were unchanged in years two to five following bronchial thermoplasty

34. Inhaber N, Goodrich R, Bolt J, M R. AQLQ Response is Correlated with a Decrease in Asthma Exacerbations in the Bronchial Thermoplasty AIR2 Population. Am J Resir Crit Care Med, 191, A4189 (2015).

35. Iyer VN, Lim KG. Bronchial thermoplasty: Reappraising the evidence (or lack thereof). Chest, 146(1), 17-21 (2014).

36. Castro M, Cox G, Wechsler ME, Niven RM. Bronchial Thermoplasty: Ready for Prime Time - The Evidence Is There! Chest, 147(2), e73-e74 (2015). 
37. Wu Q, Xing Y, Zhou X, Wang D. Meta-analysis of the Efficacy and Safety of Bronchial Thermoplasty in Patients with Moderate-to-severe Persistent Asthma. J Int Med Res, 39(1), 10-22 (2011).

38. Thomson NC, Rubin A, Niven R et al. Long term (5 Year) safety of bronchial thermoplasty: Asthma Intervention Research (AIR) trial. BMC Pulm Med, 11(1), 8 (2011).

39. Pavord ID, Thomson NC, Niven RM et al. Safety of bronchial thermoplasty in patients with severe refractory asthma. Ann Allergy Asthma Immunol, 111(5), 402-407 (2013).

40. Doeing DC, Mahajan AK, White SR, Naureckas ET, Krishnan JA, Hogarth DK. Safety and feasibility of bronchial thermoplasty in asthma patients with very severe fixed airflow obstruction: A case series. J Asthma, 50, 215-218 (2013).

41. * Bicknell S, Chaudhuri R, Lee $\mathrm{N}$ et al. Effectiveness of bronchial thermoplasty in severe asthma in 'real life' patients compared with those recruited to clinical trials in the same centre. Therap Adv Respir Dis, 9(6), 267-271 (2015).

The first observational study reporting the effectiveness and short-term safety of bronchial thermoplasty in real-life patients with severe asthma compared with those recruited to clinical trials in the same center.

42. Chakir J, Haj-Salem I, Gras D et al. Effects of Bronchial Thermoplasty on Airway Smooth Muscle and Collagen Deposition in Asthma. Annals ATS, 12(11), 1612-1618 (2015).

43. Salem IH, Boulet L-P, Biardel S et al. Long-Term Effects of Bronchial Thermoplasty on Airway Smooth Muscle and Reticular Basement Membrane Thickness in Severe Asthma. Annals ATS, 13(8), 1426-1428 (2016).

44. ** Pretolani M, Bergqvist A, Thabut $\mathrm{G}$ et al. Effectiveness of bronchial thermoplasty in patients with severe refractory asthma: clinical and histopathological correlations. $J$ Allergy Clin Immunol, 139(4), 1176-1185 (2017).

Observational study of patients with severe asthma reported improvements in clinical outcomes and reduction in airway smooth muscle mass, subepithelial basement membrane thickening, submucosal nerves, airway smooth muscle-associated nerves and epithelial neuroendocrine cells twelve months following bronchial thermoplasty. 
45. Langton D, Sha J, Ing A, Fielding D, Wood E. Bronchial thermoplasty in severe asthma in Australia. Intern Med J, 47(5), 536-541 (2017).

46. likura $\mathrm{M}$, Hojo $\mathrm{M}, \mathrm{Nagano} \mathrm{N}$ et al. Bronchial thermoplasty for severe uncontrolled asthma in Japan. Allergol Internat, (2017).

47. Zanon M, Strieder DL, Rubin AS et al. Use of MDCT to Assess the Results of Bronchial Thermoplasty. AJR Am J Roentgenol, 209(4), 752-756 (2017).

48. Kanaan R, Strange $\mathrm{C}$, Hogarth $\mathrm{K}$ et al. Bronchial Thermoplasty is Effective and Relatively Safe in High Risk Asthma Patients. Am J Respir Crit Care Med, 195, A4690 (2017).

49. Sierra M, Fernandez-Bussy S, Mehta H et al. Bronchial Thermoplasty in Severe Uncontrolled Asthma With Different Phenotypes. Chest, 152(4, Supplement), A29 (2017).

50. d'Hooghe JNS, Weersink EJM, ten Hacken NHT, Annema JT, Bonta PI. Clinical response of severe asthma patients following Bronchial Thermoplasty. Eur Respir J, 50(suppl 61), PA3029 (2017).

51. Burn J, Sims AJ, Keltie K et al. Procedural and short-term safety of bronchial thermoplasty in clinical practice: evidence from a national registry and Hospital Episode Statistics. J Asthma, 54(8), 872-879 (2017).

52. ** Chupp G, Laviolette M, Cohn L et al. Long-term outcomes of bronchial thermoplasty in subjects with severe asthma: a comparison of 3-year follow-up results from two prospective multicentre studies. Eur Respir J, 50(2) (2017).

The interim 3-year results of the PAS2 (Post-FDA Approval Clinical Trial Evaluating Bronchial Thermoplasty in Severe Persistent Asthma) study reported worse baseline characteristics and comparable clinical outcomes to the AIR2 trial

53. Debray M-P, Dombret M-C, Pretolani M et al. Early computed tomography modifications following bronchial thermoplasty in patients with severe asthma. Eur Respir J, 49(3) (2017).

54. d'Hooghe JNS, van den Berk IAH, Annema JT, Bonta PI. Acute Radiological Abnormalities after Bronchial Thermoplasty: A Prospective Cohort Trial. Respiration, 94(3), 258-262 (2017). 
55. Thomson NC, Chanez P. How effective is bronchial thermoplasty for severe asthma in clinical practice? Eur Respir J, 50(2) (2017).

56. Hanania NA, Wenzel S, Rosen K et al. Exploring the Effects of Omalizumab in Allergic Asthma. Am J Respir Crit Care Med, 187(8), 804-811 (2013).

57. Ortega HG, Liu MC, Pavord ID et al. Mepolizumab Treatment in Patients with Severe Eosinophilic Asthma. N Eng J Med, 371(13), 1198-1207 (2014).

58. Castro M, Zangrilli J, Wechsler ME, Bateman ED, Brusselle GG, Bardin P. Reslizumab for inadequately controlled asthma with elevated blood eosinophil counts: results from two multicentre, parallel, double-blind, randomised, placebo-controlled, phase 3 trials. Lancet Respir Med, 3 (2015).

59. Bleecker ER, FitzGerald JM, Chanez P et al. Efficacy and safety of benralizumab for patients with severe asthma uncontrolled with high-dosage inhaled corticosteroids and long-acting $\beta 2$-agonists (SIROCCO): a randomised, multicentre, placebo-controlled phase 3 trial. Lancet, 388(10056), 2115-2212 (2016).

60. British Guideline on the Management of Asthma. A national clinical guideline 2016. www.sign.ac.uk Accessed $4^{\text {th }}$ January 2018.

61. * Blaiss MS, Castro M, Chipps BE, Zitt M, Panettieri RA, Jr., Foggs MB. Guiding principles for use of newer biologics and bronchial thermoplasty for patients with severe asthma. Ann Allergy Asthma Immunol, 119(6), 533-540 (2017).

Report from a roundtable meeting of a panel of severe asthma experts on the definition of severe asthma, the role of biomarkers and phenotyping severe asthma, and the use of newer biologic therapies and bronchial thermoplasty to help guide practicing clinicians

62. Oberle AJ, Mathur P. Precision medicine in asthma: the role of bronchial thermoplasty. Curr Opin Pulm Med, 23(3), 254-260 (2017).

63. Zein JG, Menegay MC, Singer ME et al. Cost effectiveness of bronchial thermoplasty in patients with severe uncontrolled asthma. J Asthma, 1-7 (2015). 
64. Zafari Z, Sadatsafavi M, Marra CA, Chen W, FitzGerald JM. Cost-Effectiveness of Bronchial Thermoplasty, Omalizumab, and Standard Therapy for Moderate-to-Severe Allergic Asthma. PLoS ONE, 11(1), e0146003 (2016).

65. Nguyen HV, Bose S, Mital S et al. Is bronchial thermoplasty cost-effective as treatment for problematic asthma patients? Singapore's perspective on a global model. Respirology, 22(6), 1102-1109 (2017).

66. Niven RM, Simmonds MR, Cangelosi MJ, Tilden DP, Cottrell S, Shargill NS. Indirect comparison of bronchial thermoplasty versus omalizumab for uncontrolled severe asthma. J Asthma, 1-9 (2017).

67. Humbert M, Beasley R, Ayres J et al. Benefits of omalizumab as add-on therapy in patients with severe persistent asthma who are inadequately controlled despite best available therapy (GINA 2002 step 4 treatment): INNOVATE. Allergy, 60(3), 309-316 (2005).

68. Hanania NA, Alpan O, Hamilos DL et al. Omalizumab in Severe Allergic Asthma Inadequately Controlled With Standard Therapy. Annals of internal medicine, 154(9), 573-582 (2011).

69. Sarikonda K, Sheshadri A, Koch T et al. Predictors Of Bronchial Thermoplasty Response In Patients With Severe Refractory Asthma. Am J Respir Crit Care Med, 189, A2429 (2014).

70. Langton D, Sha J, Ing A, Fielding D, Thien F, Plummer V. Bronchial thermoplasty: activations predict response. Respir Res, 18(1), 134 (2017).

71. likura M, Ishii S, Izumi S, Yamauchi Y, Hojo M, Sugiyama H. Clinical Characteristics of Responders Treated with Bronchial Thermoplasty in Severe Asthmatic Patients. Am J Respir Crit Care Med, 195, A3208 (2017).

72. Kirby M, Ohtani K, Lopez Lisbona RM et al. Bronchial thermoplasty in asthma: 2-year follow-up using optical coherence tomography. Eur Respir J, 46(3), 859-862 (2015).

73. d'Hooghe JNS, ten Hacken NH, Weersink EJM, Sterk PJ, Annema JT, Bonta PI. Emerging understanding of the mechanism of action of Bronchial Thermoplasty in asthma. Pharmacol Therapeut, 181, 101-107 (2018). 
74. James AL, Elliot JG, Jones RL et al. Airway Smooth Muscle Hypertrophy and Hyperplasia in Asthma. Am J Respir Crit Care Med, 185(10), 1058-1064 (2012).

75. Pepe C, Foley S, Shannon J et al. Differences in airway remodeling between subjects with severe and moderate asthma. J Allergy Clin Immunol, 116(3), 544-549 (2005).

76. Solway J, Irvin CG. Airway Smooth Muscle as a Target for Asthma Therapy. N Engl J Med, 356(13), 1367-1369 (2007).

77. Zuyderduyn S, Sukkar MB, Fust A, Dhaliwal S, Burgess JK. Treating asthma means treating airway smooth muscle cells. Eur Resp J, 32(2), 265-274 (2008).

78. Gordon I, Husain A, Charbeneau J, Krishnan J, Hogarth D. Endobronchial Biopsy: A Guide for Asthma Therapy Selection in the Era of Bronchial Thermoplasty. J Asthma, 50(6), 634-641 (2013).

79. Denner DR, Doeing DC, Hogarth DK, Dugan K, Naureckas ET, White SR. Airway Inflammation after Bronchial Thermoplasty for Severe Asthma. Annals Am Thor Soc, 12(9), 1302-1309 (2015).

80. d'Hooghe JNS, ten Hacken NHT, Roelofs JJTH, Annema JT, Bonta PI. Airway smooth muscle mass reduction after Bronchial Thermoplasty; the TASMA randomized controlled trial. Eur Respir J, 50(suppl 61), PA3027 (2017).

81. Ishii S, likura M, Hojo M, Sugiyama H. Use of 3D-CT airway analysis software to assess a patient with severe persistent bronchial asthma treated with bronchial thermoplasty. Allergol Int, (2017 in press).

82. Dyrda P, Tazzeo T, DoHarris L et al. Acute Response of Airway Muscle to Extreme Temperature Includes Disruption of Actin-Myosin Interaction. Am J Respir Cell Mol Biol, 44(2), 213-221 (2011).

83. Chakir J, Haj Salem I, Gras D et al. MUC5AC expression decrease correlates with the decline in rates of severe asthma exacerbations post bronchial thermoplasty. Eur Respir J, 50(suppl 61), OA279 (2017).

84. Russell R, Singapuri A, Berair R et al. Clinical and histological effects of Bronchial Thermoplasty in severe asthma. Eur Respir J, 50(suppl 61), PA3031 (2017). 
85. Ryan DM, Fowler SJ, Niven RM. Reduction in peripheral blood eosinophil counts after bronchial thermoplasty. J Allergy Clin Immunol, 138(1), 308-310.e302 (2016).

86. Chupp G, Michaud G, Puchalski J et al. Longitudinal Effects of Bronchial Thermoplasty on Blood Gene Expression: Modulation of Inflammatory and Neuron Function. Am J Resir Crit Care Med, 191, A6431 (2015).

87. Contoli M, Santus P, Papi A. Small airway disease in asthma: pathophysiological and diagnostic considerations. Curr Opin Pulm Med, 21(1), 68-73 (2015).

88. Farah C, Langton D, Pierucci P, Ing A. Changes in Lung Function and Forced Oscillatory Technique (FOT) Parameters Following Bronchial Thermoplasty (BT) in Patients with Refractory Asthma. Am J Respir Crit Care Med, 195, A6497 (2017).

89. Baydur A, Virdi R, Barbers R. Lung Compliance and Resistance in Patients with Severe Persistent Asthma Before and After Undergoing Bronchial Thermoplasty: A Pilot Study. Am J Respir Crit Care Med, 195, A6496 (2017).

90. Boulet L-P, Laviolette M. Acute effects of bronchial thermoplasty: a matter of concern or an indicator of possible benefit to small airways? Eur Respir J, 49(3), 1700029 (2017).

91. Redberg RF. Sham Controls in Medical Device Trials. N Eng J Med, 371(10), 892-893 (2014).

92. Thomson NC. Novel approaches to the management of noneosinophilic asthma. Therap Adv Respir Dis, 10(3), 211-234 (2016).

93. Thomson NC. New and developing non-adrenoreceptor small molecule drugs for the treatment of asthma. Expert opinion on pharmacotherapy, 18(3), 283-293 (2017).

94. Pahus L, Alagha K, Sofalvi T et al. External Validity of Randomized Controlled Trials in Severe Asthma. Am J Resp Crit Care Med, 192(2), 259-261 (2015).

95. Tri JA, DeSimone CV, Daniels C et al. Electroporation ablation of bronchial smooth muscle cells: A novel non-thermal asthma therapy. Pulm Crit Care Med, 1(4), 10.15761/PCCM.1000120 (2016). 
TABLE 1: Key exclusion criteria used in the AIR, RISA or AIR2 trials of bronchial thermoplasty in asthma $[28,31,32]^{*}$

- Aged $>65$ years

- Chronic sinus disease

- Prebronchodilator $\mathrm{FEV}_{1}<60 \%$ predicted (AIR and AIR2); $\mathrm{FEV}_{1}<50 \%$ predicted (RISA trial)

- Four or more oral corticosteroid courses for asthma exacerbation within the past 12 months

- Three or more hospitalisations for asthma within the past 12 months

- Former smoker, if more than 10 pack years total smoking history

- A history of intubation for asthma, or ICU admission for asthma within the prior 24 months

- Taking maintenance oral corticosteroids $>10 \mathrm{mg}$ daily (AIR2 trial) or $>30 \mathrm{mg}$ daily (RISA trial).

Footnote

* Note: Similar key exclusion criteria were used in the PAS2 study [52] and AIR2 trial, except for chronic sinus disease. 
Table 2: Observational studies of baseline characteristics, efficacy and safety outcomes in real-life patients with severe asthma treated with bronchial thermoplasty ${ }^{+}$

\begin{tabular}{|c|c|c|c|c|c|c|c|c|c|c|}
\hline & $\begin{array}{l}\text { UK } \\
\text { [41] }\end{array}$ & $\begin{array}{l}\text { Canada } \\
{[42,43]}\end{array}$ & $\begin{array}{l}\text { France } \\
\text { [44] }\end{array}$ & $\begin{array}{c}\text { Australia } \\
\text { [45] }\end{array}$ & $\begin{array}{l}\text { Japan } \\
\text { [46] }\end{array}$ & $\begin{array}{l}\text { Brazil } \\
\text { [47] }\end{array}$ & $\begin{array}{l}\text { United States } \\
\text { and Canada } \\
\text { [52] } \\
\text { PAS2 study }\end{array}$ & $\begin{array}{c}\text { United } \\
\text { States }[48]^{+\dagger}\end{array}$ & $\begin{array}{l}\text { United } \\
\text { States } \\
{[49]^{++}}\end{array}$ & $\begin{array}{l}\text { Netherlands } \\
{[50]^{\dagger+}}\end{array}$ \\
\hline Number & 10 & 16 & 15 & 20 & 12 & 26 & 190 & 147 & 47 & 11 \\
\hline $\begin{array}{l}\text { Maintenance oral corticosteroids } \\
\text { (\%) }\end{array}$ & $40 \%$ & $30 \%$ & $66 \%$ & $50 \%$ & $42 \%$ & NR & $19 \%$ & $\begin{array}{c}29 \% \\
\text { (>10 mg } \\
\text { daily) }\end{array}$ & $47 \%$ & NR \\
\hline $\begin{array}{l}\text { FEV }_{1} \text { percent predicted (range or } \\
\text { SD) }\end{array}$ & $\begin{array}{c}72 \%(45- \\
96 \%)\end{array}$ & $\begin{array}{c}67 \%(42- \\
103 \%)\end{array}$ & $71 \%(17)$ & $63 \%(33-95 \%)$ & $71 \%(22)$ & $78 \%(16)$ & $85 \%(13)$ & $\begin{array}{l}<60 \% \text { in } 51 \% \\
\text { of patients }\end{array}$ & $63 \%$ & NR \\
\hline $\begin{array}{l}\text { Time post-bronchial } \\
\text { thermoplasty when clinical } \\
\text { outcomes assessed, months }\end{array}$ & 12 & $\begin{array}{c}12[42] \\
(n=9, \\
\geq 27)[43]\end{array}$ & 12 & 6 & 12 & 12 & 36 & 12 & 12 & 6 \\
\hline Asthma control score(s) & $\begin{array}{l}\text { Improved } \\
(40 \%)^{*}\end{array}$ & Improved & Improved & $\begin{array}{l}\text { Improved } \\
(85 \%)^{*}\end{array}$ & $\begin{array}{l}\text { Improved } \\
(50 \%)^{*}\end{array}$ & NR & NR & NR & NR & Improved \\
\hline
\end{tabular}




\begin{tabular}{|c|c|c|c|c|c|c|c|c|c|c|}
\hline Asthma quality of life score & $\begin{array}{c}\text { Improved } \\
(50 \%)^{*}\end{array}$ & NR & Improved & & $\begin{array}{c}\text { Improved } \\
(67 \%)^{*}\end{array}$ & $\begin{array}{c}\text { Improved } \\
(65 \%)^{*}\end{array}$ & NR & NR & NR & Improved \\
\hline Severe exacerbations & $\begin{array}{c}\text { Decreased } \\
(30 \%)^{*}\end{array}$ & Decreased & Decreased & Decreased & $\begin{array}{c}\text { Decreased } \\
(83 \%)\end{array}$ & NR & Decreased & $\begin{array}{c}\text { Decreased } \\
\text { (trend) }\end{array}$ & Decreased & $\begin{array}{c}\text { Decreased } \\
(82 \%)\end{array}$ \\
\hline ED visits or hospital admissions & $\begin{array}{c}\text { Decreased } \\
(30 \%)^{*}\end{array}$ & NR & Decreased & NR & Decreased & NR & Decreased & Decreased & Decreased & NR \\
\hline Daily oral corticosteroid dose & $\begin{array}{c}\text { Decreased } \\
(n=1)\end{array}$ & $\begin{array}{c}\text { Decreased } \\
\qquad(n=4)\end{array}$ & Decreased & Decreased & $\begin{array}{c}\text { Decreased } \\
(n=1)\end{array}$ & NR & Decreased & Decreased & NR & NR \\
\hline $\mathrm{FEV}_{1}$ & No change & No change & $\begin{array}{c}\text { No } \\
\text { change }\end{array}$ & $\begin{array}{c}\text { No change } \\
\text { overall } \\
\text { (Increased if } \\
\mathrm{FEV}_{1}<60 \% \text { ) }\end{array}$ & Increased & No change & No change & No change & No change & NR \\
\hline $\begin{array}{l}\text { Assessment of overall beneficial } \\
\text { response to bronchial } \\
\text { thermoplasty during the post- } \\
\text { treatment period (percent) }\end{array}$ & $50 \%$ & NR & $73 \%$ & $65-85 \%$ & $>80 \%$ & $65 \%$ & NR & NR & NR & $55 \%$ \\
\hline $\begin{array}{l}\text { Number of hospital admissions } \\
\text { due to asthma during to the peri- } \\
\text { procedure treatment period }\end{array}$ & $\begin{array}{c}\mathbf{3}(\mathbf{3} \\
\text { patients) }\end{array}$ & $\begin{array}{c}6 \text { (2 } \\
\text { patients) }\end{array}$ & NR & 2 (1 patient) & NR & NR & $13.2 \%$ & $21 \%$ & NR & NR \\
\hline
\end{tabular}


+ Observational studies reporting results of bronchial thermoplasty from 10 or more patients with severe asthma.

${ }^{+\dagger}$ Preliminary reports published as abstracts.

Abbreviations: $\mathrm{FEV}_{1}$, forced expiratory volume in one second; ED, emergency department; NR, not reported; PAS2, Post-FDA Approval Clinical Trial Evaluating Bronchial Thermoplasty in Severe Persistent Asthma

\# Assess using different asthma symptom questionnaire: asthma control questionnaire[41,45,50]; asthma control test [44]; asthma control scoring system $[42,43]$

* Percent of patients with $\geq$ minimal clinical important difference (MCID) in clinical outcome 
FIGURE LEGENDS

Figure 1

Title: (A) Total Asthma Quality of Life Questionnaire (AQLQ) score over 12 months after treatment with bronchial thermoplasty and (B) healthcare utilization events during the post-treatment period.

(a) Change in Asthma Quality of Life Questionnaire (AQLQ) score over 12 months after treatment with bronchial thermoplasty (BT) (diamonds) or sham control (squares) in the per protocol population. ${ }^{*}$ Posterior probability of superiority $=97.9 \%$.

(b) Severe exacerbations (exacerbation requiring treatment with systemic corticosteroids or doubling of the inhaled corticosteroids dose), emergency department visits, and hospitalizations occurring in the post-treatment period. Open bars, sham; shaded bars, bronchial thermoplasty. All values are means \pm SEM. *Posterior probability of superiority $=$ 95.5\%. †Posterior probability of superiority = 99.9\%.'

Reprinted with permission of the American Thoracic Society. Copyright (C) 2017 American Thoracic Society. [28]. The American Journal of Respiratory and Critical Care Medicine is an official journal of the American Thoracic Society. 
Figure 2

Title: Severe exacerbations and Emergency Department visits in the 5 years after bronchial thermoplasty

A, Proportion of subjects with severe exacerbations. B, Severe exacerbation rates. C, Proportion of subjects with emergency department (ED) visits for respiratory symptoms. D, ED visit rates. Values are point estimates with 95\% upper and lower Cls. The 365-day period constituting year 1 began at 6 weeks after the last bronchial thermoplasty bronchoscopy. Reprinted with permission [33]

Figure 3

Title: Structural effects of bronchial thermoplasty in bronchial biopsy specimens from patients with severe asthma.

'Bright-field micrographs of bronchial biopsy specimens subjected to quadruple immunohistochemical staining for smooth muscle actin (red), the vascular endothelial marker CD31 (green), lymph endothelial marker, podoplanin (D2-40, brown), and the neuronal marker PGP.9.5 (black) are shown. A and B, Biopsy specimens taken before and 3 months after bronchial thermoplasty, respectively (note that Fig 2, B, rather than representing the average, exemplifies a case in which smooth muscle was virtually absent). C, A neuroendocrine cell (NEC) in the bronchial epithelium detected based on nuclear distribution of PGP (arrowhead). D and E, Subepithelial nerves (arrowheads) in the subepithelial region before (Fig 2, D) and after (Fig 2, E) bronchial thermoplasty. F, Smooth muscle-associated nerves (arrowhead) exemplified in a biopsy specimen collected before bronchial 
thermoplasty treatment. Scale bars $5250 \mathrm{~mm}$ (Fig 2, A and B) or $40 \mathrm{~mm}$ (Fig 2, C-F). sm, Smooth muscle; bv, blood vessels; Iv, lymphatic vessels'.

Reprinted with permission [44]

\section{Figure 4}

Title: Algorithm to guide the selection of add-on treatment with biologics and bronchial thermoplasty for patients with severe refractory asthma*.

* Patients with severe asthma (step 5) who have persistent symptoms and exacerbations should be assessed by a specialist in severe asthma to confirm the correct diagnosis and to address aggravating factors, such as non-adherence, poor inhaler technique and comorbidities. Add-on treatment with biologics and bronchial thermoplasty are considered for patients with uncontrolled asthma despite high dose ICS plus LABA and the long-acting muscarinic antagonist tiotropium. 